Ophthalmologe 2012 · 109:110-111

DOI 10.1007/s00347-011-2451-5

๑) Springer-Verlag 2012
P. Charbel Issa ${ }^{1,2}$

${ }^{1}$ Universitäts-Augenklinik Bonn

${ }^{2}$ Oxford Eye Hospital and Nuffield Laboratory of Ophthalmology, University of Oxford

\title{
Therapiekonzepte bei erblichen Netzhautdystrophien
}

Erbliche Netzhautdystrophien sind eine heterogene Gruppe von Erkrankungen, die schon in frühem Lebensalter zur Erblindung führen können. In der Altersgruppe zwischen 21 und 60 Jahren ist diese Erkrankungsgruppe die häufigste Ursache für Erblindung oder schweren Sehverlust [1]. Das therapeutische Vorgehen begrenzte sich lange auf unterstützende Maßnahmen wie den Ausgleich von Refraktionsfehlern, die Anpassung von Sehhilfen, die Optimierung der Umgebungsbedingungen sowie die genetische Beratung [3]. Abgesehen von 3 seltenen Krankheitsbildern (Refsum-Syndrom, Abetalipoproteinämie, Atrophia gyrata), bei denen diätetische Maßnahmen den Krankheitsverlauf verbessern, konnte der eigentliche Erkrankungsverlauf erblicher Netzhautdystrophien bisher jedoch kaum aktiv beeinflusst werden.

Fortschritte in der molekulargenetischen Diagnostik erlauben zunehmend, erbliche Netzhautdystrophien gemäß ihren genetischen Ursachen zu unterscheiden. So sind derzeit 175 Gene identifiziert, deren Mutation zur Entwicklung einer Netzhautdystrophie führen kann (https://sph.uth.tmc.edu/retnet). Darüber hinaus ermöglichen neue hochauflösende Methoden der retinalen Bildgebung und Funktionsdiagnostik eine immer detailliertere phänotypische Differenzierung der unterschiedlichen Erkrankungsbilder. Jedoch blieben diese diagnostischen Fortschritte zunächst weiterhin ohne direkten therapeutischen Nutzen für betroffene Patienten.

Durch interdisziplinäre Zusammenarbeit finden zunehmend innovative The- rapieansätze zur Behandlung von Patienten mit erblichen Netzhautdystrophien den Weg aus dem Labor in klinische Studien. Diese Ausgabe von Der Ophthalmologe soll einen Überblick über derzeitige Konzepte und neue Entwicklungen bei der Therapie erblicher Netzhautdystrophien bieten.

Grundsätzlich wird die Wahl des therapeutischen Vorgehens bei Patienten mit Netzhautdystrophien von der klinischen Ausgangssituation abhängig sein (- Tab. 1). So wird nach Verlust der Photorezeptoren versucht, deren Funktion zu ersetzen, wohingegen bei noch vorhandenen Photorezeptoren darauf abgezielt wird, deren Zelltod zu verhindern oder hinauszuzögern. Neben diesen Ansätzen zur Behandlung der eigentlichen Photorezeptordegeneration wird häufig auch die Therapie von assoziierten okulären Veränderungen notwendig. Auch hier steht eine Reihe von Therapieoptionen zur Verfügung, wie beispielhaft in - Tab. 2 aufgezeigt.

\section{Pharmakologische und gentherapeutische Therapieoptionen}

Der erste Beitrag befasst sich mit pharmakologischen Therapiemöglichkeiten, die neuerdings als Neuroprotektiva zur Verlangsamung des Zelltods und zur Korrektur spezieller Dysfunktionen (z. B. 9-cisRetinal) erprobt werden. Solche Therapieansätze beruhen häufig auf einem verbesserten Verständnis der Pathophysiologie erblicher Netzhautdystrophien. Pharmakologische Ansätze, mit Netzhautdystrophien assoziierte Veränderungen wie ein
Makulaödem oder eine chorioidale Neovaskularisation $\mathrm{zu}$ behandeln, werden ebenfalls besprochen.

Im zweiten Beitrag wird das Konzept der retinalen Gentherapie vorgestellt, die in unterschiedlicher Form - abhängig von der klinischen Ausgangssituation - zum Einsatz kommen kann. Aufgrund der genetischen Ursache erblicher Netzhautdystrophien kann die retinale Gentherapie potenziell eine ursächliche Therapie darstellen (spezifische Gentherapie). Darüber hinaus können gentherapeutische Techniken aber auch eingesetzt werden, um photosensitive Ionenkanäle (sog. Optogenetik) oder neuroprotektive Faktoren in der Netzhaut zu exprimieren (unspezifische Gentherapie).

\section{Elektrostimulation und retinale Implantate}

Im dritten Beitrag wird die transkorneale Elektrostimulation vorgestellt. Bei dieser Methode werden schwache elektrische Ströme mittels kornealer Elektroden appliziert. Es wird davon ausgegangen, dass unter bestimmten Stimulationsbedingungen neuroprotektive Faktoren in der Netzhaut freigesetzt werden. Diese könnten eine Verlangsamung des Zelltods bewirken.

Schließlich wird in einem vierten Beitrag erläutert, was ehemals erblindete $\mathrm{Pa}$ tienten mit subretinalem Implantat im Alltag sehen können. Retinale Implantate haben in letzter Zeit viel Aufsehen erregt, da hiermit erstmals in klinischen Studien ein Funktionsgewinn bei zuvor erblindeten Patienten erreicht wurde (z. B. $[4,5])$. 
Tab. 1 Mögliche therapeutische Ansätze bei erblichen Netzhautdystrophien in Abhängigkeit von der klinischen Ausgangssituation. (Adaptiert nach [2])

\begin{tabular}{lll}
\hline $\begin{array}{l}\text { Klinische Aus- } \\
\text { gangssituation }\end{array}$ & $\begin{array}{l}\text { Therapeutischer } \\
\text { Ansatz }\end{array}$ & Beispiele \\
\hline $\begin{array}{l}\text { Noch vorhan- } \\
\text { dene Photore- } \\
\text { zeptoren }\end{array}$ & $\begin{array}{l}\text { Korrektur der Dys- } \\
\text { funktion }\end{array}$ & $\begin{array}{l}\text { Spezifische Gentherapie } \\
\text { Pharmakologisch }\end{array}$ \\
\cline { 2 - 3 } & $\begin{array}{l}\text { Verlangsamung des } \\
\text { Zelltods }\end{array}$ & $\begin{array}{l}\text { Neuroprotektion (unspezifische Gentherapie, pharma- } \\
\text { kologisch, diätetisch, zellbasiert, Elektrostimulation) } \\
\text { Vermeidung potenzieller Noxen (z. B. Rauchen, Licht) } \\
\text { Eventuell Nahrungsergänzungsmittel }\end{array}$ \\
\cline { 2 - 3 } & $\begin{array}{l}\text { Spezielle diätetische } \\
\text { Maßnahmen }\end{array}$ & $\begin{array}{l}\text { Refsum-Syndrom } \\
\text { Abetalipoproteinämie } \\
\text { Atrophia gyrata }\end{array}$ \\
\hline $\begin{array}{l}\text { Nach Verlust } \\
\text { der Photorezep- } \\
\text { toren }\end{array}$ & $\begin{array}{l}\text { Ersatz für Photorezep- } \\
\text { torfunktion }\end{array}$ & $\begin{array}{l}\text { Retinales Implantat } \\
\text { Optogenetik }\end{array}$ \\
\cline { 2 - 3 } & $\begin{array}{l}\text { Erneuerung von Photo- } \\
\text { rezeptoren und/oder re- } \\
\text { tinalem Pigmentepithel }\end{array}$ & $\begin{array}{l}\text { Photorezeptortransplantation } \\
\text { Stammzelltherapie }\end{array}$ \\
\hline Manche Ansätze, wie z. B. die Photorezeptortransplantation, werden bisher nicht in klinischen Studien getestet. \\
\hline
\end{tabular}

Tab. 2 Beispiele für Therapieoptionen zur Behandlung okulärer Veränderungen, die in Assoziation mit erblichen Netzhautdystrophien auftreten können

\begin{tabular}{ll}
\hline Komplikation & Therapie \\
\hline Makulaödem & Versuch mit Acetazolamid, ggf. titrierte Dauertherapie \\
\hline Chorioidale Neovaskularisation & Anti-VEGF-Therapie, photodynamische Therapie \\
\hline Katarakt & Linsenersatz \\
\hline Gefäßektasien & Gegebenenfalls Laserkoagulation oder Kryotherapie \\
\hline VEGF „Vascular endothelial growth factor”. & \\
\hline
\end{tabular}

\section{Ist eine molekulargenetische Diagnose notwendig?}

Die spezifische Gentherapie, bestimmte Pharmakotherapien (z. B. 9-cis-Retinal) und spezielle diätetische Maßnahmen sind nur bei exakter, idealerweise molekulargenetisch gesicherter Diagnose sinnvoll, da sie nur bei bestimmten genetischen Ursachen wirken können. Dies erklärt, dass bei Anwendung solcher maßgeschneiderter Therapien in Zukunft eine molekulargenetische Sicherung der klinischen Diagnose in vielen Fällen notwendig wird.

Dagegen wirken unspezifische Therapieansätze wie die Neuroprotektion oder retinale Implantate potenziell unabhängig vom Genotyp. Jedoch muss beachtet werden, dass der Effekt von ehemals als unspezifisch angesehenen Therapieansätzen möglicherweise doch vom zugrunde liegenden molekulargenetischen Defekt abhängig ist. Im Extremfall könnte eine Therapie sogar eine negative Auswirkung bei bestimmten genetischen Veränderungen zur Folge haben (beispielsweise Vi- tamin A bei abca4-Mutationen). Insofern ist unter Umständen auch bei derzeit als unspezifisch angesehenen Therapien eine molekulargenetische Untersuchung hilfreich.

\section{Herausforderungen bei Therapiestudien zu erblichen Netzhautdystrophien}

Die Etablierung neuer Therapien für Patienten mit Netzhautdystrophie geht mit einer Vielzahl von Herausforderungen einher. Bei den oft langsam fortschreitenden Erkrankungen ist die Messung eines Therapieerfolges schwierig. Dies ist insbesondere bei einer systemischen Therapie der Fall, da dann kein Auge als interne Kontrolle dienen kann. Aufgrund einer hohen interindividuellen Variabilität und möglicherweise unterschiedlicher Wirkung in Abhängigkeit vom Erkrankungsstadium wären größere Patientenkohorten notwendig, die aber bei den zumeist seltenen Erkrankungen nicht vorhanden sind. Auch kann von einem Therapieerfolg in einem Knock-out-Mausmodel nicht zwangsläufig auf eine gleiche Wirkung bei Patienten mit Mutation im selben Gen geschlossen werden.

\section{Fazit}

Dieser Überblick soll aufzeigen, dass Patienten mit Netzhautdystrophien zunehmend Therapieoptionen angeboten werden können. Für die korrekte Anwendung sind häufig eine exakte morphologische und molekulargenetische Diagnostik notwendig. Die meisten Therapien werden derzeit noch in Studien getestet, in denen sie sich zunächst bewähren müssen. Deshalb sollte eine Behandlung in Zentren erfolgen, um die Therapieeffekte weiter einer wissenschaftlichen Auswertung zugänglich zu machen. Nur so können wir in Zukunft Therapien mit gesichertem Nutzen empfehlen.

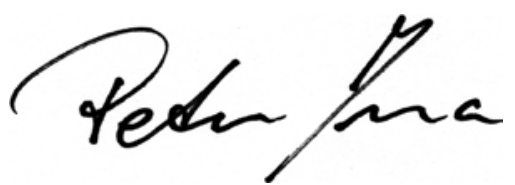

Korrespondenzadresse

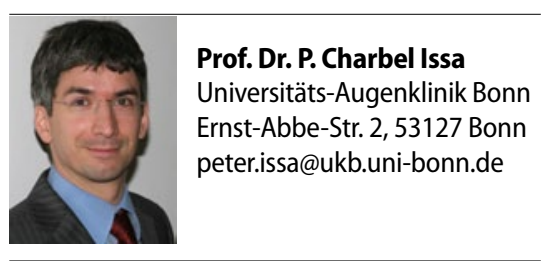

Danksagung. Gefördert durch die ProRetina.

\section{Literatur}

1. Finger RP, Fimmers R, Holz FG et al (2011) Prevalence and causes of registered blindness in the largest federal state of Germany. Br J Ophthalmol 95:1061-1067

2. Jacobson SG, Cideciyan AV (2010) Treatment possibilities for retinitis pigmentosa. N Engl J Med 363:1669-1671

3. Kellner U, Tillack H, Renner AB (2004) Hereditäre Netzhaut-Aderhaut-Dystrophien, Teil 1: Pathogenese, Diagnostik, Therapie, Patientenbetreuung. Ophthalmologe 101:307-319

4. Zrenner E, Bartz-Schmidt KU, Benav H et al (2011) Subretinal electronic chips allow blind patients to read letters and combine them to words. Proc Biol Sci 278:1489-1497

5. Weiland JD, Cho AK, Humayun MS (2011) Retinal prostheses: current clinical results and future needs. Ophthalmology 118:2227-2237 